\title{
A fuzzy solution approach for a multi-objective integrated production-distribution model with multi products and multi periods under uncertainty
}

\author{
A. Gharehyakheh ${ }^{\mathbf{a}^{*}}$ and R. Tavakkoli-Moghaddam ${ }^{\mathrm{b}}$
}

${ }^{a}$ Department of Economic Engineering, University of Economic Science, Tehran, Iran

${ }^{b}$ Department of Industrial Engineering, College of Engineering, University of Tehran, Tehran, Iran

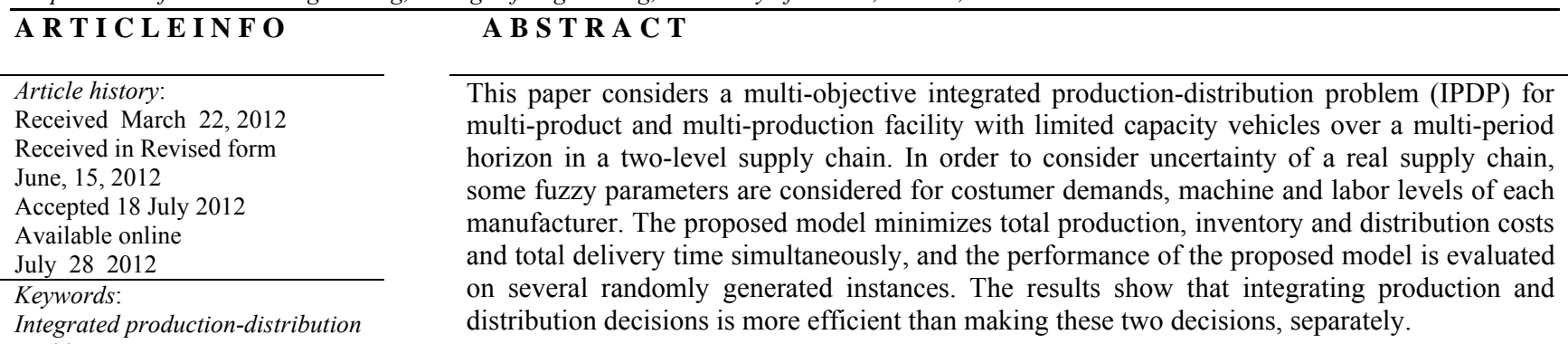

problem

Fuzzy parameters

Uncertainty

\section{Introduction}

Supply chains are generally viewed as a network of materials and information flows among different facilities, including manufacturing and assembly plants and distribution centers (Thomas, \& Griffin, 1996; Sabri \& Beamon, 2000). Distribution costs often represent a significant fraction of the actual cost of a finished product. In some sectors, they even dominate production costs. Ideally, this is why any cost reduction effort should encompass production planning and distribution planning (Boudia et al., 2007). It is a challenging problem for manufacturers to optimize their supply chain (Bard \& Nananukul, 2010). A vehicle routing problem (VRP), for instance, is deeply analyzed (Toth \& Vigo, 2002). For even stochastic model, there are many studies (Gendreau et al., 1996). A few studies include inventory constraints (Christiansen \& Nygreen, 1998; Dror \& Ball, 1987). However, there are few studies that contain both production and distribution decisions (Zubair, 1999; Vidal \& Goetschalckx, 1997). On the other hand, production planning has been studied widely and deeply

* Corresponding author.

E-mail addresses: gharehyakheh@ses.ac.ir (A. Gharehyakheh) 
without enough consideration on distribution part (Mula et al., 2006). Bhatnagar and Chandra (1993) classified general coordination research into three categories, each representing attempts to coordinate different operations of the firm. These categories represent an integration of decision making as (1) supply and production planning, (2) production and distribution planning, and (3) inventory and distribution planning, respectively.

To make production and distribution decisions in a supply chain, there are two approaches, namely (1) solving production and distribution problems separately and (2) integrating production and distribution problems. The first approach is widely investigated in the literature. In recent studies, the integration of these two main supply chain problems is developed (Bard \& Nananukul, 2010; Armentano et al., 2011; Bauso et al., 2010; Bilge, 2010; Shiguemoto \& Armentano, 2010). Fig. 1 shows a sample of production and distribution problem. In this study, we compare these two approaches and represent the associated results.

Note that the considered problem belongs to a class of NP-hard ones and it is reduced to the VRP, which is a well-known NP-hard problem, in a single period case (Boudia et al., 2007). Lei et al. (2006) formulated the production, inventory and distribution routing problem as a mixed-integer model. The main advantage lies in its ability to simultaneously coordinate the production, inventory and transportation operations of the entire planning horizon, without any need to aggregate the demand or relax constraints on transportation capacities. Boudia et al. (2007) developed a similar production, distribution problem for one product over a multi-period horizon and used the GRASP method with path relinking. Boudia and Prins (2009) used a memetic algorithm with population management to solve the model and compared the results with a two-phase heuristic and GRASP.

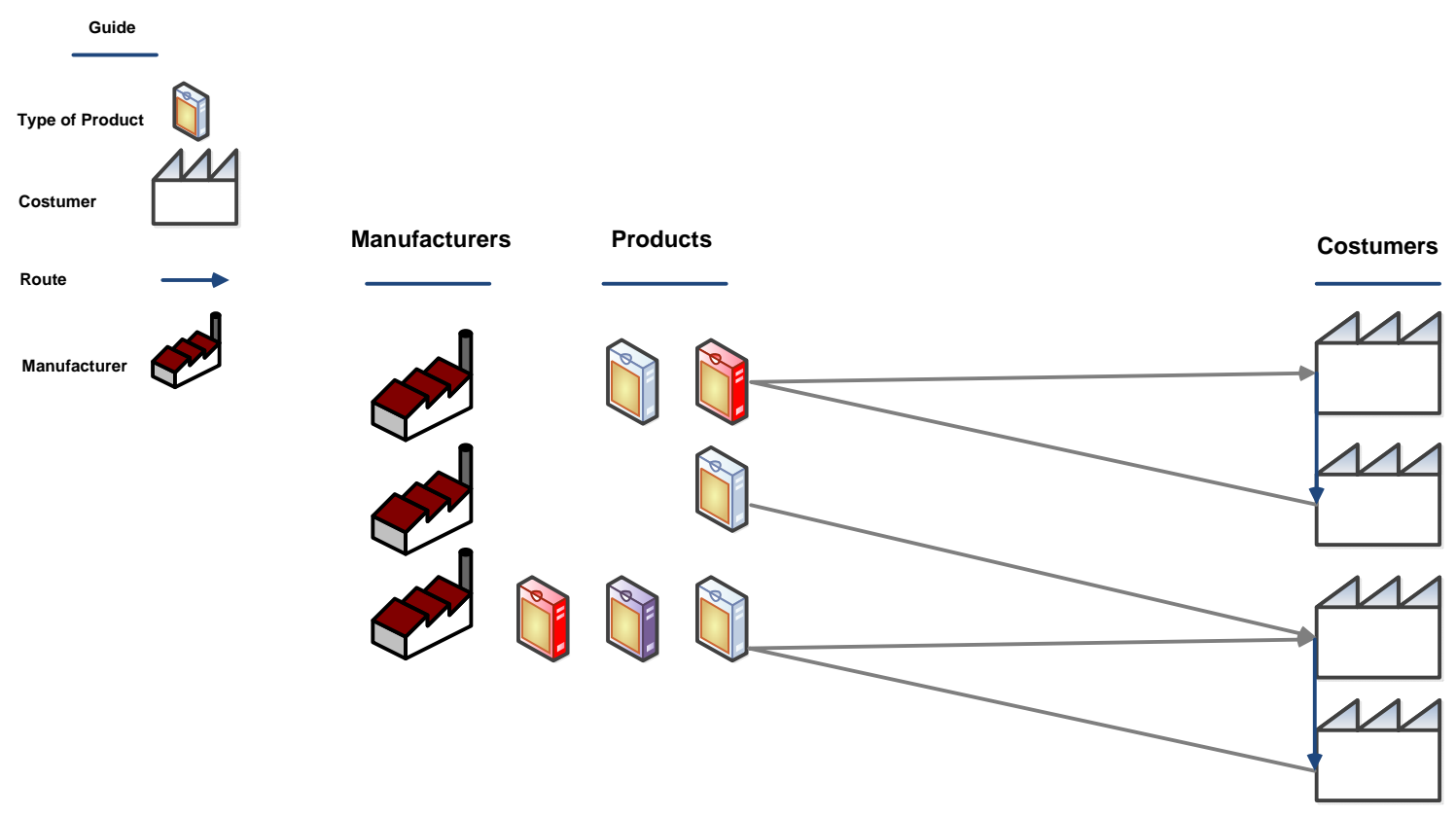

Fig. 1. A production and distribution model

Dealing with real-world situations requires assuming more actual assumptions on the model. In this study, the presented model considers uncertainty in the costumers demand and machine and labor level at manufacturers as fuzzy parameters. There are some studies to consider the decision makers' interest or assume uncertainty as fuzzy parameters. Selim et al. (2008) developed a multi-objective linear programming model. In order to reflect the collaborative planning issues to their model and to provide a more realistic model structure, decision makers' imprecise aspiration levels for the goals are incorporated into the model using a fuzzy goal programming approach. Aliev et al. (2007) developed a fuzzy integrated multi-period and multi-product production and distribution model since there is a need for a joint general strategic plan for production and distribution model in a supply chain. In the 
current study, our proposed fuzzy parameters and objective functions are very close to Liang (2008, 2011). The main differences are the formulation of objective functions and the approach of involving fuzzy parameters in the model.

The remainder of this paper is as follows. Section 2 explains the problem statement and develops the mixed-integer linear model. A defuzzification procedure and aggregation of objective functions are represented in Section 3. Section 4 contains computational experiments and sensitivity analysis. Finally, Section 5 presents the conclusion.

\section{Problem statement}

In this section, a new mixed-integer linear programming (MILP) model is presented to find out the best strategy for production, inventory levels and distribution, simultaneously.

\subsection{Model assumptions}

The following assumptions are considered in the presented model.

- Each manufacturer can produce all types of products.

- Delivery time and capacity of each vehicle are limited.

- Time limitation of the vehicle is less than a time period and costumers receive distributed products of each period on the same period.

- Vehicles load products from one manufacturer and distribute them between one or more costumers and vehicles cannot visit more than one manufacturer at each period.

- Storage capacity at each facility is limited.

- Costumers' demand, machine and labor levels at each manufacturer assume as fuzzy parameters.

- All the vehicles are available at each period.

- Shortage is not allowed, costumers demand must be supplied.

\subsection{Notations and parameters}

In the development of the model, these following notations are used.

$j \quad$ Set of products

$i \quad$ Set of manufacturers

$k \quad$ Set of costumers

$w \quad$ Set of manufacturers and costumers

$v \quad$ Set of homogeneous vehicles

$t \quad$ Set of time periods parameters

$C_{i j}^{p} \quad$ Production cost for each production unit of product $j$ at manufacturer $i$

$h_{i j}{ }^{s} \quad$ Unit holding cost of product $j$ at manufacturer $i$

$h_{k j}{ }^{c} \quad$ Unit holding cost of product $j$ at costumer $k$

$f_{i j}^{p} \quad$ Production setup cost of product $j$ at manufacturer $i$

$c_{w k} \quad$ Distribution cost between node $w$ to $k$

$t_{l w k} \quad$ Time that vehicles spend to travel from node $w$ to $k$ (hour)

$\widetilde{D}_{j k t} \quad$ Fuzzy demand of product $j$ from costumer $k$ at time period $t$ (unit)

$l_{i j} \quad$ Hour of labor per unit to produce product $j$ at manufacturer $i$ (man-hour/unit)

$\tilde{L}_{i} \quad$ Fuzzy maximum labor levels available on manufacturer $i$ (man-hour)

$r_{i j} \quad$ Hour of machine per unit to produce product $\mathrm{j}$ at manufacturer $i$ (machine-hour/unit)

$\widetilde{M}_{i} \quad$ Fuzzy maximum machine levels available on manufacturer $i$ (machine-hour) 
$U_{i} \quad$ Maximum inventory that can be held by manufacturer $i$

$U_{k} \quad$ Maximum inventory that can be held by costumer $k$

$M_{1} \quad$ Large enough number

$M_{2} \quad$ Large enough number

Cap Capacity of vehicles

$P_{t} \quad$ Length of time period(hours)

\subsection{Decision variables}

$P_{i j t} \quad$ Production quantity of product $j$ at manufacturer $i$ on time period $t$

$I_{i j t} \quad$ Inventory level of product $j$ at manufacturer $i$ on time period $t$

$I_{k j t} \quad$ Inventory level of product $j$ at costumer $k$ on time period $t$

$R_{j k t} \quad$ Total amount of product $j$ delivered to costumer $k$ on time period $t$

$y_{i j t} \quad 1$ if manufacturer in produce product $j$ on the time period $t ; 0$ otherwise

$x_{w j k t}{ }^{v} \quad$ Total amount of product $j$ transported from node $w$ to $k$ by vehicle $v$ on time period $t$

$z_{w k t}{ }^{v} \quad 1$ if vehicle $v$ travel from node $w$ to $k$ on time period $t ; 0$ otherwise.

\subsection{Basic model}

Based on the above-mentioned notations and variables, the model for the integrated productiondistribution problem (IPDP) is formulated as follows.

$\min z_{1}=\sum_{t=1}^{T} \sum_{i=1}^{I} \sum_{j=1}^{J} p_{i j t} C_{i j}^{p}+\sum_{t=1}^{T} \sum_{i=1}^{I} \sum_{j=1}^{J} I_{i j t} h_{i j}^{S}+\sum_{t=1}^{T} \sum_{k=1}^{K} \sum_{j=1}^{J} I_{k j t} h_{k j}^{c}+$

$\sum_{t=1}^{T} \sum_{i=1}^{I} \sum_{j=1}^{J} Y_{i j t} f_{i j}^{p}+\sum_{v=1}^{V} \sum_{w=1}^{W} \sum_{k=1}^{K} \sum_{t=1}^{T} Z_{w k t}^{v} C_{w k}^{v}$

$\min \mathrm{z}_{2}=\sum_{v=1}^{V} \sum_{w=1}^{W} \sum_{k=1}^{K} \sum_{t=1}^{T} t l_{w k} Z_{w k t}^{v}$

subject to

$$
\begin{array}{lc}
\sum_{i=1}^{I} P_{i j t}+\sum_{i=1}^{I} I_{i j(t-1)}-\sum_{i=1}^{I} I_{i j t}=\sum_{k=1}^{K} R_{j k t} & ; \forall t, j \\
R_{j k t}+I_{j k t}-I_{j k(t-1)}=\widetilde{D}_{j t k} & ; \forall t, j, k \\
l_{i j} P_{i j t} \leq \tilde{L}_{i} & ; \forall i, j, t \\
r_{i j} P_{i j t} \leq \widetilde{M}_{i} & ; \forall i, j, t \\
P_{i j t} \leq M_{1} Y_{i j t} & ; \forall i, j, t \\
\sum_{v=1}^{V} \sum_{w=1}^{W} x_{w j k t}^{v}-\sum_{v=1}^{V} \sum_{w=1}^{W} x_{k j k t}^{v}=R_{j k t} & ; \quad \forall, j, k \\
\sum_{w=1}^{W} \sum_{k=1}^{K} t l_{w k} Z_{w k t}^{v} \leq P t & \\
\sum_{j=1}^{J} x_{w j k t}^{v} \leq M_{2} Z_{w k t}^{v} & ; \forall t, v
\end{array}
$$


$\sum_{k=1}^{K} Z_{w k t}^{v} \leq 1$

$; \forall t, v, w$

$w_{1} \neq w_{2}, k, \sum_{w_{1}=1}^{W} Z_{w_{1} k t}^{v}-\sum_{w_{2}=1}^{W} Z_{k w_{2} t}^{v}=0 \quad ; \forall t, v$

$0 \leq \sum_{j} I_{j w t} \leq U_{w} \quad ; \forall w, t$

$P_{i j t} \geq 0, R_{j k t} \geq 0, x_{w j k t}^{v} \geq 0, I_{j w t} \geq 0, Y_{i j t}=(0,1), Z_{w k t}^{v}=(0,1) ; \forall i, j, k, w, v, t$

The first objective function (1) attempts to reduce the total cost including setup and production costs at manufacturer inventory costs at both manufacturer and costumers and distribution costs. The second objective function (2) attempts to minimize the total delivery times. Constraint (3) ensures the equilibrium of produced and stored products and delivered products. Constraint (4) represents fuzzy demands of costumers are supplied by delivering products and inventories. Constraints (5) and (6) show that manufacturer cannot produce more than their resource. Constraint (7) shows the setup cost when production happens. Constraint (8) shows that a delivery may happen to or from a costumer but the net delivered products are constant. Constraint (9) shows the total delivery time of each vehicle must be less than period time. Constraint (10) ensures that if there is a delivery between two routes, distribution cost should be considered. Constraint (11) shows that each vehicle cannot carry more than their capacity. Constraint (12) considers that each vehicle cannot visit more than one costumer the same time. Constraint (13) ensures that all vehicles must distribute the products in a cycle graph. Constraint (14) considers an upper bound for inventory at each facility. Constraint (15) defines types of each variable.

\section{Solution procedure}

To solve the proposed IPDP model, first, fuzzy constraints convert to the equivalent crisp constraints, then a fuzzy solution approach uses to solve the model.

\subsection{Equivalent crisp constraints}

In this research, the proposed MILP model with fuzzy parameters is converted into an equivalent auxiliary crisp model by applying an efficient method by hybridizing the novel methods of Jimenez et al. (2007). In the literature, several methods have been developed to deal with parameters (e.g., Jiménez, 2007; Lai, \& Hwang, 1993; Wang \& Liang, 2005; Inuiguchi \& Ramík, 2000; Arenas Parra et al., 2005). We use Pishvaee and Torabi's method (2010), which is based on a close-loop supply chain network design model. The proposed model uses a hybridizing based on Jimenez et al. (2007) and Parra et al. (2005). We use this method to convert the proposed fuzzy mixed-integer linear model into an equivalent auxiliary crisp model. As Pishvaee and Torabi (2010) discussed, it is based on the Jimenez's method (2007) because of its computational efficiency and using for different kinds of membership functions.

There are some recent studies in supply chain that Jimenez's method (2007) is used (e.g, Pishvaee \& Torabi, 2010; Peidro et al., 2010). The method is based on 'expected value' and 'expected interval' of 
a fuzzy number. Let $\tilde{c}$ be a triangular fuzzy number (see Fig. 2), in which the following equation can be defined as a membership function of $\tilde{c}$.

$$
\mu_{\tilde{c}}(x)=\left\{\begin{array}{rrr}
f_{c}(x)=\frac{x-c^{p}}{c^{m}-c^{p}} & \text { if } c^{p} \leq x \leq c^{m} \\
1 & \text { if } x=c^{m} \\
g_{c}(x)=\frac{c^{o}-x}{c^{o}-c^{m}} & \text { if } c^{m} \leq x \leq c^{o} \\
0 & \text { if } x \leq c^{p} \text { or } x \geq c^{o}
\end{array}\right.
$$

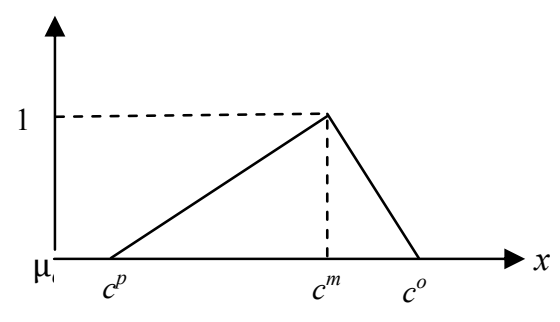

Fig. 2. Fuzzy triangular membership function

According to Jimenez (1996), for triangular fuzzy number $\tilde{c}$, we can define the expected value (EV) and expected interval (EI) as follows:

$$
\begin{aligned}
& E V(\tilde{c})=\frac{E_{1}^{c}-E_{2}^{c}}{2}=\frac{c^{p}+2 c^{m}+c^{o}}{4} \\
& E I(\tilde{c})=\left[E_{1}^{c}, E_{2}^{c}\right]=\left[\int_{0}^{1} f_{c}^{-1}(x) d x, \int_{0}^{1} g_{c}^{-1}(x) d x\right]=\left[\frac{\left(c^{p}+c^{m}\right)}{2}, \frac{\left(c^{m}+c^{o}\right)}{2}\right]
\end{aligned}
$$

The same equations can be used for a trapezoidal fuzzy numbers. According to the ranking method of Jimenez (2007), Eq. (19) can be defined for any pair of fuzzy numbers $\tilde{a}$ and $\tilde{b}$ where the degree $\tilde{a}$ is bigger than $\tilde{b}$.

$$
\mu_{M}(\tilde{a}, \tilde{b})=\left\{\begin{array}{cc}
1 & \text { if } E_{1}^{a}-E_{2}^{b}>0 \\
\frac{E_{2}^{a}-E_{1}^{b}}{\left(E_{2}^{a}-E_{1}^{b}\right)-\left(E_{1}^{a}-E_{2}^{b}\right)} & \text { if } 0 \in\left[E_{1}^{a}-E_{2}^{b}, E_{2}^{a}-E_{1}^{b}\right] \\
0 & \text { if } E_{2}^{a}-E_{1}^{b}<0
\end{array}\right.
$$

$\mu_{M}(\tilde{a}, \tilde{b}) \leq \alpha$ shows that $\tilde{a}$ is smaller than or equal to $\tilde{b}$ at least in degree of $\alpha$, this is represented as $\tilde{a} \leq_{\alpha} \tilde{b}$. In proposed model, the fuzzy constraints can be formulated in general by:

$\tilde{a} x \leq \tilde{b}, x \geq 0$

According to Eq. (19), the equation $\tilde{a} x \leq \tilde{b}$ can be written as follow:

$$
\frac{E_{2}^{a x}-E_{1}^{b}}{E_{2}^{a x}-E_{1}^{a x}+E_{2}^{b}-E_{1}^{b}} \leq \alpha
$$

The above equation can be written as follow:

$$
\left[(1-\alpha) E_{2}^{a}+\alpha E_{1}^{a}\right] x \leq \alpha E_{2}^{b}+(1-\alpha) E_{1}^{b}
$$

According to above, proposed fuzzy constraints of IPDP can be written as equal crisp form: 
$R_{j k t}+I_{j k t}-I_{j k(t-1)}=\alpha\left(\frac{D_{j k t}^{p}+D_{j k t}^{m}}{2}\right)+(1-\alpha)\left(\frac{D_{j k t}^{m}+D_{j k t}^{o}}{2}\right) \quad ; \forall j, k, t$

$l_{i j} P_{i j t} \leq \alpha\left(\frac{L_{i}^{p}+L_{i}^{m}}{2}\right)+(1-\alpha)\left(\frac{L_{i}^{m}+L_{i}^{o}}{2}\right)$

; $\forall i, j, t$

$r_{i j} P_{i j t} \leq \alpha\left(\frac{M_{i}^{p}+M_{i}^{m}}{2}\right)+(1-\alpha)\left(\frac{M_{i}^{m}+M_{i}^{o}}{2}\right) \quad ; \forall i, j, t$

\subsection{Fuzzy solution approach}

These two objective functions are aggregated as an aggregate objective function, in which the weighted-compromise function is used.

$\left.\begin{array}{l}\operatorname{Min} z_{1}=\mu_{1} \\ \operatorname{Min} z_{2}=\mu_{2}\end{array}\right\} \operatorname{Min} z=\mu_{1}^{S}+b \mu_{2}^{S}$

According to the Messac et al. (2007), parameters $b>0$ and $s$ are positive integers. This aggregate objective function is chosen because it offers the required flexibility to change its curvature and orientation by adjusting the free parameters for capturing Pareto-optimal points. Additionally, it is locally admissible in the first quadrant and globally admissible for $s$ odd. $b$ adjusts the units of two objective functions and applies interest of the decision makers. For $s=1$, the aggregate objective function becomes the weighted-sum of the objectives, and other Pareto-optimal corner points lie on the concave part of the Pareto frontier and cannot be captured. In this paper, the proposed IPDP model is solved by combination of Jimenez et al. (2007), Parra et al. (2005), Pishvaee and Torabi (2010) and Messac et al. (2000). This Method is represented by the following steps:

Step 1: Determine the appropriate triangular possibility distributions for fuzzy parameters, and formulate the multi-objective MILP model for the IPDP problem.

Step 2: Determine the minimum acceptable feasibility degree of decision vector $\alpha$, convert the fuzzy constraints into crisp ones, and formulate the equivalent auxiliary crisp multi-objective MILP model.

Step 3: Convert multi-objective functions into a single-objective function using the aggregation function presented in Messac et al. (2000).

Step 4: Solve the single objective MILP model. If the decision maker is satisfied with the current solution, stop the algorithm; otherwise, provide another solution by changing the value of $b$ and $\alpha$, and then go to Step 2.

\section{Computational results}

Several experiments are implemented to show the validity of the proposed model. In this section, two test problems are designed to compare the IPDP model results with the classic model. Table 1 shows the size of the test problems.

\section{Table 1}

Size of test problems

\begin{tabular}{ccccc}
\hline Problem no. & No. of manufacturers & No. of products & No. of costumers & No. of Time periods \\
\hline 1 & 2 & 2 & 2 & 4 \\
2 & 4 & 2 & 3 & 3 \\
\hline
\end{tabular}


According to Pishvaee and Torabi (2010), the three prominent points (i.e., the most likely, the most pessimistic and the most optimistic values) are estimated for each triangular fuzzy parameters, and two random numbers $r_{1}$ and $r_{2}$ are generated between 0.2 and 0.8 by using a uniform distribution, and the most pessimistic $\left(c^{p}\right)$ and optimistic $\left(c^{o}\right)$ values of fuzzy number $(\tilde{c})$ are calculated as follows:

$c^{o}=\left(1+r_{1}\right) * c^{m}$

$c^{p}=\left(1-r_{2}\right) * c^{m}$

To compare the classic approach with the proposed IPDP model, both models are coded in the GAMS optimization software. The experiments have been performed on Pentium Dual at $2.66 \mathrm{GHz}$ having 4 GB of RAM. The value of $b$ sets is set to 500 because the value can balance the essence of two objectives. These two models are compared in different $\alpha$ levels. These parameters are set for three test problems and the results can be illustrated in Table 2. As the results shows, if the decision maker uses the IPDP model instead of the classic model, its integrated objective will be improved and the CPU time will decrease.

To show how the objective functions are sensitive to parameter $b$, these two models are tested according to the different values of $b$ at $\alpha$-level 0.5 (see Table 3). As it can be seen from the results, the solution procedure used for both models has diverse solutions. Whereby, the proposed solution method is sensitive to the value of parameter $b$. Finally, it can be concluded that IPDP model is more appropriate making production and distribution decisions in a supply chain.

Table 2

Summary of the test results according to different $\alpha$-levels.

\begin{tabular}{|c|c|c|c|c|c|}
\hline \multirow[b]{2}{*}{ Problem no. } & \multirow[b]{2}{*}{$\alpha$-level } & \multicolumn{2}{|c|}{ IPDP model } & \multicolumn{2}{|c|}{ Classic model } \\
\hline & & $\begin{array}{c}\text { Integrated } \\
\text { OFV }\end{array}$ & $\begin{array}{l}\text { Elapsed run } \\
\text { time (s) }\end{array}$ & $\begin{array}{c}\text { Integrated } \\
\text { OFV }\end{array}$ & $\begin{array}{c}\text { Elapsed run } \\
\text { time (s) }\end{array}$ \\
\hline \multirow{9}{*}{1} & 0.1 & 117539 & 0.241 & 130446 & 0.846 \\
\hline & 0.2 & 112859 & 0.813 & 125226 & 0.892 \\
\hline & 0.3 & 108119 & 0.719 & 119916 & 0.938 \\
\hline & 0.4 & 103379 & 0.775 & 114626 & 0.842 \\
\hline & 0.5 & 98879 & 0.839 & 101670 & 0.890 \\
\hline & 0.6 & 94019 & 0.902 & 104146 & 0.920 \\
\hline & 0.7 & 89549 & 0.890 & 92080 & 0.855 \\
\hline & 0.8 & 84689 & 0.789 & 87060 & 0.879 \\
\hline & 0.9 & 79799 & 0.802 & 87348 & 0.905 \\
\hline \multirow{9}{*}{2} & 0.1 & 100330 & 2.175 & 133255 & 1000 \\
\hline & 0.2 & 96549 & 1.715 & 128115 & 596 \\
\hline & 0.3 & 92617 & 1.653 & 122772 & 1000 \\
\hline & 0.4 & 88994 & 1.025 & 117582 & 559 \\
\hline & 0.5 & 85100 & 1.690 & 112332 & 1000 \\
\hline & 0.6 & 81454 & 1.499 & 107172 & 1000 \\
\hline & 0.7 & 77667 & 1.087 & 101982 & 1000 \\
\hline & 0.8 & 73856 & 1.676 & 96612 & 1000 \\
\hline & 0.9 & 70107 & 1.043 & 91569 & 1000 \\
\hline
\end{tabular}


Table 3

Summary of the results on the sensitivity analysis of $b$

\begin{tabular}{cccccc}
\hline \multirow{2}{*}{$\alpha$-level } & $\begin{array}{c}\text { Different value of } \\
b\end{array}$ & $\begin{array}{c}\text { Integrated } \\
\text { OFV }\end{array}$ & $\begin{array}{c}\text { Elapsed run } \\
\text { time (s) }\end{array}$ & $\begin{array}{c}\text { Integrated } \\
\text { OFV }\end{array}$ & $\begin{array}{c}\text { Elapsed run } \\
\text { time (s) }\end{array}$ \\
\cline { 2 - 5 } & 5 & 84649 & 1.640 & 111725 & 1000 \\
0.05 & 100 & 84737 & 1.119 & 111844 & 1000 \\
& 500 & 85100 & 1.651 & 112332 & 1000 \\
& 6000 & 90654 & 1.657 & 119042 & 1001 \\
& 100000 & 164288 & 1.639 & 202628 & 7.735 \\
\hline
\end{tabular}

\section{Conclusion}

In this study, an integrated production-distribution problem (IPDP) has been considered under fuzzy demands of costumers as well as machine and labor levels of manufacturers. A mixed-integer linear programming (MILP) model has been represented for the IPDP model. To solve the presented model, a hybrid fuzzy solution method has been proposed. This solution method can take the decision makers' preferences into account. Combination of production and distribution problems has been considered in recent years, and according to the literature, formulating an IPDP model under uncertainty is still scars. This results in having a more efficient supply chain. The results of the IPDP and classical models with different $\alpha$-level and $b$ values have been compared in terms of objective function values and CPU times. For future research, many other studies can develop the IPDP model for a three level supply chain that includes distribution centers among the production facilities and costumers. Moreover, uncertainty can be considered in other parameters, such as delivery times for vehicles. Developing a heuristic or meta-heuristic algorithm is needed for solving large-size problems.

\section{References}

Aliev, R.A., Fazlollahi, B., Guirimov, B.G., \& Aliev, R.R. (2007). Fuzzy-genetic approach to aggregate production-distribution planning in supply chain management. Information Sciences, 177(20), 42414255.

Arenas Parra, M., Bilbao Terol, A., Pérez Gladish, B., \& Rodríguez Uría, M.V. (2005). Solving a multiobjective possibilistic problem through compromise programming. European Journal of Operational Research, 164(3), 748-759.

Armentano, V.A., Shiguemoto, A.L., \& Løkketangen, A. (2011). Tabu search with path relinking for an integrated production-distribution problem. Computers \& Operations Research, 38(8), 1199-1209.

Bard, J.F., \& N. Nananukul, A. (2010). Branch-and-price algorithm for an integrated production and inventory routing problem. Computers \& Operations Research, 37(12), 2202-2217.

Bauso, D., Blanchini, F., \& Pesenti, R. (2010). Average Flow Constraints and Stabilizability in Uncertain Production-Distribution Systems. Journal of Optimization Theory and Applications, 144(1), 12-28.

Bhatnagar, R., Chandra, P., \& Goyal, S.K. (1993). Models for multi-plant coordination. European Journal of Operational Research, 67(2), 141-160.

Bilge, B. (2010). Application of fuzzy mathematical programming approach to the production allocation and distribution supply chain network problem. Expert Systems with Applications, 37(6), 4488-4495.

Boudia, M., \& Prins, C. (2009). A memetic algorithm with dynamic population management for an integrated production-distribution problem. European Journal of Operational Research, 195(3), 703715. 
Boudia, M., Louly, M.A.O., Prins, C. (2007). A reactive GRASP and path relinking for a combined production-distribution problem. Computers \& Operations Research, 34(11), 3402-3419.

Christiansen, M., \& Nygreen, B. (1998). A method for solving ship routing problems with inventory constraints. Annals of Operations Research, 81(0), 357-378.

Dror, M., \& Ball, M. (1987). Inventory/routing: Reduction from an annual to a short-period problem. Naval Research Logistics, 34(6), 891-905.

Gendreau, M., Laporte, G., \& Séguin, R. (1996). Stochastic vehicle routing. European Journal of Operational Research, 88(1), 3-12.

Inuiguchi, M., \& Rami'k, J. (2000). Possibilistic linear programming: a brief review of fuzzy mathematical programming and a comparison with stochastic programming in portfolio selection problem. Fuzzy Sets and Systems, 111(1), 3-28.

Jiménez, M., Arenas, M., Bilbao, A., \& Victoria Rodríguez, M. (2007). Linear programming with fuzzy parameters: An interactive method resolution. European Journal of Operational Research, 177(3), 1599-1609.

Jiménez, M. (1996). Ranking fuzzy numbers through the comparison of its expected intervals. International Journal of Uncertainty, Fuzziness and Knowledge-Based Systems, 4(4), 379-388.

Lai, Y.-J., \& Hwang, C.L. (1993). Possibilistic linear programming for managing interest rate risk. Fuzzy Sets and Systems, 54(2), 135-146.

Lei, L., Liu, S., Ruszczynski, A., \& Park, S. (2006). On the integrated production, inventory, and distribution routing problem. IIE Transactions, 38(11), 955-970.

Messac, A., Puemi-Sukam, C., \& Melachrinoudis, E. (2000). Aggregate Objective Functions and Pareto Frontiers: Required Relationships and Practical Implications. Optimization and Engineering, 1(2), 171188.

Mula, J., Poler, R., García-Sabater, J.P., \& Lario, F.C. (2006). Models for production planning under uncertainty: A review. International Journal of Production Economics, 103(1), 271-285.

Peidro, D., Mula, J., Jiménez, M., del Mar Botella, M. (2010). A fuzzy linear programming based approach for tactical supply chain planning in an uncertainty environment. European Journal of Operational Research, 205(1), 65-80.

Pishvaee, M.S., \& Torabi, S.A. (2010). A possibilistic programming approach for closed-loop supply chain network design under uncertainty. Fuzzy Sets and Systems, 161(20), 2668-2683.

Sabri, E.H., \& Beamon, B.M. (2000). A multi-objective approach to simultaneous strategic and operational planning in supply chain design. Omega, 28(5), 581-598.

Selim, H., Araz, C., \& Ozkarahan, I. (2008). Collaborative production-distribution planning in supply chain: A fuzzy goal programming approach. Transportation Research Part E: Logistics and Transportation Review, 44(3), 396-419.

Shiguemoto, A.L., \& Armentano, V.A. (2010). A tabu search procedure for coordinating production, inventory and distribution routing problems. International Transactions in Operational Research, 17(2), 179-195.

Thomas, D.J., \& Griffin, P.M. (1996). Coordinated supply chain management. European Journal of Operational Research, 94(1), 1-15.

Tien-Fu, L. (2011). Application of fuzzy sets to manufacturing/distribution planning decisions in supply chains. Information Sciences, 181(4), 842-854.

Tien-Fu, L. (2008). Fuzzy multi-objective production/distribution planning decisions with multi-product and multi-time period in a supply chain. Computers \& Industrial Engineering, 55(3), 676-694.

Toth, P., \& Vigo, D. (2002). Models, relaxations and exact approaches for the capacitated vehicle routing problem. Discrete Applied Mathematics, 123(1-3), 487-512.

Vidal, C.J., \& Goetschalckx, M. (1997). Strategic production-distribution models: A critical review with emphasis on global supply chain models. European Journal of Operational Research, 98(1), 1-18.

Wang, R.-C., \& Liang, T.-F. (2005). Applying possibilistic linear programming to aggregate production planning. International Journal of Production Economics, 98(3), 328-341.

Zubair, M.M. (1999). An integrated production-distribution model for a multi-national company operating under varying exchange rates. International Journal of Production Economics, 58(1), 81-92. 